\title{
Panorama epidemiológico del tabaquismo en México
}

\author{
Roberto Tapia-C onyer, MD, MPH, MS, (1) Pablo Kuri-Morales, MD, MSc, ${ }^{(2)}$ \\ María Jesús Hoy-Gutiérrez, MD.(2)
}

\section{Tapia-Conyer R, Kuri-Morales $\mathbf{P}$, Hoy-Gutiérrez MJ. \\ Panorama epidemiológico del tabaquismo en México. \\ Salud Publica Mex 2001;43:478-484. \\ El texto completo en inglés de este artículo está disponible en: http://www.insp.mx/salud/index.html}

\section{Resumen}

El tabaco es una de las principales causas prevenibles de enfermedad y muerte en el mundo: 3.5 millones de personas fumadoras fallecen anualmente. El presente ensayo muestra los avances de esta epidemia en nuestro país y señala la importancia de aplicar un programa integral que permita detener su avance. Se analizan y comparan los resultados de las tres Encuestas $N$ acionales de Adicciones hechas en México en 1988, 1993 y 1998, y se concluye que así como las estrategias se han enfocado hacia un programa integral que contempla acciones de prevención, protección de los no fumadores, cesación, control de la publicidad e incremento de impuestos, disminuir el consumo y los daños asociados a la salud depende de todos. El texto completo en inglés de este artículo está disponible en:http://www.insp.mx/ salud/index.html

Palabras clave: tabaquismo; prevalencia; México

\author{
Tapia-Conyer R, Kuri-Morales $\mathbf{P}$, \\ Hoy-Gutiérrez MJ. \\ An Epidemiologic Overview \\ of Smoking in Mexico. \\ Salud Publica Mex 2001;43:478-484. \\ The English version of this paper \\ is available at: http://www.insp.mx/salud/index.html
}

\section{Abstract}

Smoking is one of the main preventable causes of disease and death worldwide; 3.5 million smokers die annually. This essay shows the progress made against this epidemic in our country and points out the relevance of implementing a comprehensive program to control smoking. Results from three national surveys conducted in Mexico in 1988, 1993, and 1998 are analyzed and compared. $0 \mathrm{n}$ the one hand, the strategies should include preventive actions, protection of non-smokers, smoking cessation, banning of smoking advertisement, and tax increases; on the other hand, controlling smoking and its consequences on health depend on all of us. The English version of this paper is available at: http:// www.insp.mx/salud/index.html

Key words: smoking; prevalence; Mexico
E 1 tabaco es una de las principales causas preveniE bles de enfermedad y muerte en el mundo, además de ser una sustancia adictiva, socialmente aceptada y de consumo legal. La Organización Mundial de la Salud (OMS) señala que de la población mundial 30\% de los adultos son fumadores, y de estos fumadores 3.5 millones fallecen al año, lo que equivale a la muerte de siete personas cada minuto por enfermedades relacionadas con el tabaquismo. ${ }^{1,2}$ Sin embargo, al triplicarse en China el consumo de tabaco durante 20

(1) Subsecretaría de Prevención y Control de Enfermedades, Secretaría de Salud, México.

(2) Dirección General de Epidemiología, Secretaría de Salud, México.

(3) Dirección General de Epidemiología, Secretaría de Salud, México.

Fecha de recibido: 22 de noviembre de 2000 - Fecha de aprobado: 14 de febrero de 2001 Solicitud de sobretiros: Dr. Pablo Kuri Morales. Francisco P. Miranda N 0. 177, 40 Piso, colonia Unidad Lomas de Plateros, 01480 México, D.F., México. Correo electrónico: pkuri@ epi.org.mx 
años, aumentó considerablemente la prevalencia de tabaquismo y la mortalidad atribuible. ${ }^{3}$ En México, el consumo per cápita ha disminuido de 1501 cigarrillos anuales en 1970 a 754 cigarrillos para 1997; sin embargo, estimaciones conservadoras indican que en nuestro país mueren aproximadamente 122 personas por día debido a enfermedades asociadas con el tabaquismo; en total, más de 44 mil fumadores mueren al año.

A pesar de que en la actualidad se conocen los daños a la salud causados por fumar, el tabaquismo continúa en ascenso, especialmente en los países en desarrollo; por las proporciones alcanzadas se le considera una epidemia universal. Globalmente, hay casi 1100 millones de fumadores, de los cuales 300 son de países desarrollados (relación de dos hombres por cada mujer), en comparación con los 800 en países en desarrollo (siete hombres por cada mujer). Estos datos apoyan la teoría de que la epidemia del tabaquismo ha llegado a su máximo punto en países desarrollados, pero se encuentra en ascenso en los países en desarrollo. Proyecciones de la OMS indican que, si continúa el consumo actual, para el año 2020 habrá 10 millones de muertes anuales por enfermedades relacionadas con el consumo de tabaco, de las cuales siete de cada diez ocurrirán en países en desarrollo. ${ }^{1}$ Además, los países en desarrollo, como México, serán el blanco de las estrategias de las industrias tabacaleras transnacionales en busca de nuevos mercados de potenciales fumadores, en particular, entre los jóvenes y las mujeres. ${ }^{4}$

\section{Prevalencia del tabaquismo en México}

Los primeros estudios realizados en México para conocer la prevalencia de fumadores iniciaron hace más de 20 años; sin embargo, la mayoría se llevaron a cabo en grupos de poblaciones heterogéneas o con metodología diferente, dificultando la comparación e identificación de tendencias en el ámbito nacional. ${ }^{5} \mathrm{La}$ Dirección General de Epidemiología (DGE) y el Instituto Mexicano de Psiquiatría (IMP), de la Secretaría de Salud, realizaron la primera Encuesta Nacional de Adicciones en 1988 (ENA-1988) cuya metodología, adaptada del esquema propuesto por la OMS en 1980, permitió conocer la prevalencia y distribución del consumo de tabaco en la población urbana nacional de 12 a 65 años de edad. En 1993 se realizó la segunda Encuesta Nacional de Adicciones (ENA-1993), y para 1998 se llevó a cabo la tercera Encuesta Nacional de Adicciones (ENA-1998), con el propósito de conocer más a fondo esta problemática y así mejorar las estrategias de prevención y control del tabaquismo en México.

La prevalencia de fumadores obtenida en las tres encuestas nacionales muestra un discreto incremento.
En 1988, la prevalencia global de la población urbana entre los 12 y 65 años de edad, fue de $25.8 \%$, mientras que en 1993 fue de $25.1 \%$, incrementándose en 1998 a $27.7 \%$. En cuanto a la prevalencia por sexo, ésta permaneció relativamente similar en las tres encuestas donde los hombres presentaron el mayor porcentaje de consumo. Por lo que respecta a los ex fumadores, se observó un descenso que va de $21.6 \%$ en la encuesta de 1988, a 20.3\% en 1993, hasta $14.8 \%$ en 1998, y para los nunca fumadores muestra un ascenso de $52.6 \%$ (ENA) 1988, 54.6\% (ENA-1993), hasta 57.4\% (ENA-1998) (figura 1). ${ }^{6}$

Durante 1988 más de 9 millones de mexicanos eran fumadores activos, la mayoría hombres [6549,400 hombres $(71 \%)$ versus 2724,600 mujeres $(29 \%)]$. Hacia 1998, estas cifras se incrementaron a más de $13 \mathrm{mi}-$ llones de fumadores con una proporción de $67 \%$ hombres y 33\% mujeres. En 1988 la mayoría de los fumadores tenían entre 18 y 29 años de edad; para 1998 este mismo grupo de edad también presentó el mayor porcentaje de consumo (38.1\%).

En cuanto a la frecuencia, $64 \%$ fumaba diariamente en 1988; en 1993 disminuyó a 62\% y para 1998 se conservó dicha tendencia a la baja con $52 \%$. De los que fuman a diario, 49\% en 1988 y 1993 eran fumadores leves ( 1 a 5 cigarros), mientras que en 1998 se observó un incremento a $74 \%$, una diferencia importante al comparar estas cifras con las de otros países con mayor consumo de cigarros y mayor potencial de adicción a la nicotina. ${ }^{6}$

Algunos de los fumadores actuales comenzaron a fumar antes de los 18 años, aun cuando está prohibida legalmente la venta de tabaco a menores de edad. De aproximadamente $52 \%$ en 1988 , la proporción de fumadores actuales que empezaron a fumar antes de los 18 años se incrementó a 61.4\% para 1998, lo que apoya la teoría de que después de la adoles-

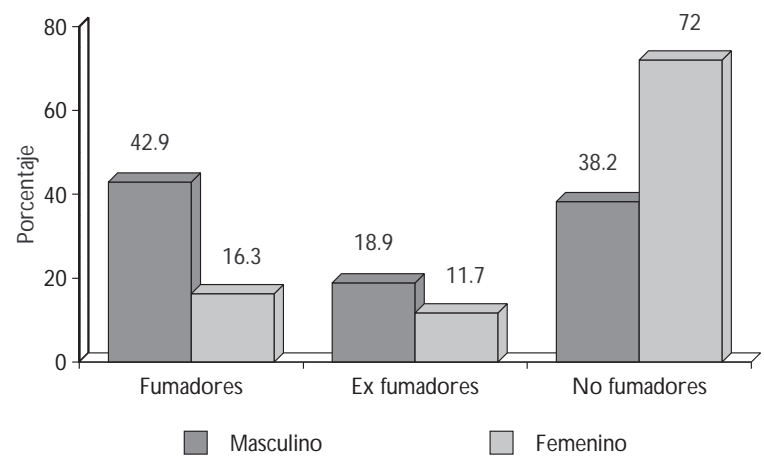

Figura 1. Prevalencia de tabaquismo según patrón de Consumo de tabaco y POR SeXo. MéXico, 1998 
cencia, si una persona no ha comenzado a fumar, aparentemente disminuye la probabilidad de que lo haga (en 1988 sólo $7.6 \%$ empezaron a fumar después de los 26 años, en 1993 7.4\% y 5.5\% en 1998). Además, se ha observado que los motivos sociales tienen un gran peso en el inicio del tabaquismo: ocho de cada diez fumadores se iniciaron principalmente por la curiosidad y la presión de los amigos.

\section{$\mathrm{N}$ iños y adolescentes: grupos de alto riesgo}

Algunos de los factores que contribuyen a que los niños $y$ adolescentes sean un grupo de riesgo para el consumo de tabaco incluyen, entre otros, la facilidad de acceso a los cigarros, la presión de grupo y la promoción de tabaco.

En México se ha identificado que el tabaquismo entre los niños y los adolescentes es un problema en ascenso. Asimismo, se observó en las Encuestas Nacionales que la prevalencia de fumadores adolescentes se incrementó de $7.7 \%$ en 1988, a 10.1\% en la de 1993, y para la última, 1998, de $11.6 \%$, lo que demuestra un incremento de $51 \%$ en los últimos 10 años, a pesar de que la Ley General de Salud prohíbe desde 1984 la venta de cigarros a menores de edad. De los fumadores entre 12 y 17 años de edad, se observa que aumenta el consumo de tabaco entre la primaria y la secundaria (20\% versus $43 \%$, respectivamente).

Además, la Encuesta Nacional sobre el Uso de Drogas entre la Comunidad Escolar de 1992, realizada por la Secretaría de Educación Pública (SEP) y el Instituto Mexicano de Psiquiatría (IMP), señala que tres de cada diez adolescentes, entre los 13 y 18 años, admiten haber fumado alguna vez, a pesar de que $89.3 \%$ consideran que fumar una o más cajetillas al día es peligroso y 73.2\% consideran que sus amigos no lo aceptarían. En dicho estudio se señala que entre los 13 y 14 años fue la edad en la cual la mayoría comenzó a fumar. En cuanto al género, $38.1 \%$ de los jóvenes y $20.2 \%$ de las jóvenes fumaron alguna vez. ${ }^{7}$

Con el propósito de evaluar el porcentaje de expendedores de tabaco a menores de edad, y para comprobar la facilidad de compra de los adolescentes, en 1997 la DGE llevó a cabo una encuesta de accesibilidad a los cigarros en las 16 delegaciones del Distrito Federal y se comprobó que $79 \%$ de las tiendas visitadas les vendió cigarros; sólo 1\% de los expendedores preguntó la edad al menor y $12 \%$ de las tiendas tenían señalamiento de la prohibición de la venta de tabaco a menores de edad, lo cual no se relacionó con la no venta del producto al menor. ${ }^{8}$ Resultados similares se obtuvieron en otro estudio realizado en
1999 en Ciudad Juárez, Chihuahua, donde se encontró que en $98 \%$ de las tiendas se vendieron cigarros a menores de edad. ${ }^{9}$

Como se observó en la ENA-1988, la presión de grupo, así como de los amigos, contribuyó a que $34.6 \%$ de los fumadores empezaran con esta adicción, incrementándose a 39.0\% para 1993.

Estos datos concuerdan con los de la encuesta de la SEP y el IMP, en donde los estudiantes señalan que más de la mitad de sus amigos fuman cigarros. De la encuesta de 1988, 77\% opinó que la promoción de cigarros influye para que la gente fume.

El tabaquismo en los adolescentes representa un problema serio, ya que invita al consumo de otras drogas: se asocia a un mayor riesgo $(14 \%)$ de consumo de mariguana y alcohol. ${ }^{6}$ Además, $97.2 \%$ de los fumadores consumen una segunda droga, principalmente alcohol, y el $83 \%$ consume una tercera: la mariguana. ${ }^{10}$

Resulta prioritario señalar que para lograr un impacto significativo en la reducción del tabaquismo en los adolescentes, se requiere mayor cumplimiento de la legislación vigente, que prohíbe la venta de tabaco a menores de edad, para disminuir el acceso, sensibilizar a los expendedores y a la población sobre esta problemática, así como una regulación estricta de la promoción del tabaco para que ésta no se dirija a menores. ${ }^{11}$

\section{Prevalencia de fumadores involuntarios}

Actualmente no existe duda alguna de que el tabaquismo causa daños en la salud de los fumadores activos. Sin embargo, en los últimos años el tabaquismo también se ha asociado en los fumadores pasivos como un factor de riesgo de enfermedades relacionadas con su consumo. Un fumador pasivo es aquel no fumador (ex fumador o nunca fumador) que se expone involuntariamente al humo del tabaco, especialmente en un ambiente cerrado.

El humo del tabaco en el ambiente se deriva de la denominada fuente principal (fumador activo) y la colateral (aerosol de la combustión del tabaco). De esta última se deriva casi $85 \%$ del humo en un espacio cerrado, con una composición similar a la que se exponen los fumadores activos. Aunque el humo colateral se diluye en el aire la exposición es constante, además de mantener niveles elevados de carcinógenos y agentes tóxicos. Por lo tanto, se ha observado que el humo del tabaco incrementa el riesgo de daños a la salud del fumador involuntario, por ejemplo cáncer pulmonar e infarto agudo del miocardio, infecciones respiratorias y asma, éstas, especialmente en los niños de padres fumadores. 
En 1988, 42.5\% de la población encuestada se componía de fumadores involuntarios expuestos en su casa (11 358400 personas), a pesar de que $87.4 \%$ consideró que fumar afecta la salud de los demás.

Cinco años más tarde, $41 \%$ de la población eran fumadores involuntarios (34000 000 personas); 29\% ex fumadores y $71 \%$ nunca fumadores. De éstos, $31 \%$ eran menores de edad y la mayoría de los fumadores involuntarios fueron mujeres. Para 1998 los fumadores involuntarios se incrementaron a $52.6 \%$ de la población (18 144 032), prevaleciendo como tales las mujeres con 61.3\% (11 114 294).

En el caso de los ex fumadores notamos un decremento de los mismos pues para 1988 representaban 21.6\% (7 792 056). Y para 1993 encontramos 20.3\% (8 615 731), y se observó una disminución de los mismos para 1998, donde representaban 14.8\% (7 081 404) de la población encuestada.

El principal lugar de exposición al humo del tabaco sigue siendo la casa, lo que conlleva la necesidad urgente de fomentar la educación en la familia para reducir el número de fumadores que exponen a sus miembros y a un conocido agente nocivo. ${ }^{6}$

Hoy hay aproximadamente 31 millones de personas expuestas a los daños a la salud causados por el tabaco, lo que representa poco menos de la tercera parte de la población total de México.

\section{Mortalidad por enfermedades asociadas al consumo de tabaco}

La enfermedad isquémica del corazón y los tumores malignos de pulmón, bronquios y tráquea ocuparon los primeros lugares en la mortalidad general en $1998 .{ }^{12}$ La tasa de mortalidad por cáncer pulmonar es más elevada en los hombres, un reflejo del mayor consumo en este grupo, y desde 1985 hasta 1998 se ha incrementado en más de $20 \%$ en ambos sexos (figura 2).

Se estima que en México más de 44 mil mexicanos murieron en 1998 por enfermedades relacionadas con el consumo de tabaco, lo que equivale, utilizando la fracción atribuible estimada por el Departamento de Salud y Servicios Humanos de los Estados Unidos, para cada padecimiento relacionado con este hábito, publicada en el reporte de 1992, Tabaco o Salud: Situación de las Américas de OPS/OMS, ${ }^{2}$ a que 122 mexicanos murieron diariamente (figura 3). Estas muertes representan el 10\% de las muertes nacionales. En un estudio hecho en 1992, se compararon los datos de 1970, 1980 y 1990, y se observó un incremento en las tasas de mortalidad de las principales causas de muerte relacionadas con el tabaco. ${ }^{13}$ La tasa de cáncer pulmonar aumentó $220 \%$ y la enfermedad coronaria

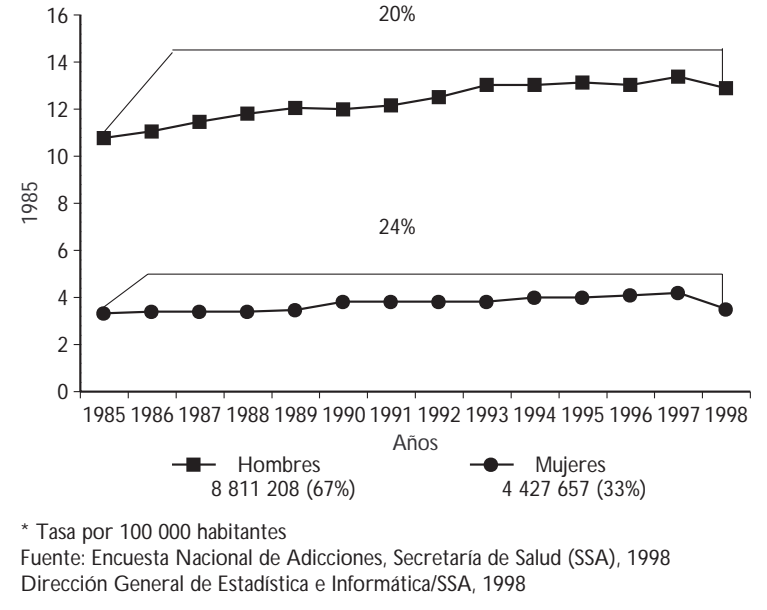

FIGURA 2.T ABAQUISMO Y TENDENCIA DE MORTALIDAD POR CÁNCER DE PULMÓN. MÉXICO, 1985-1998

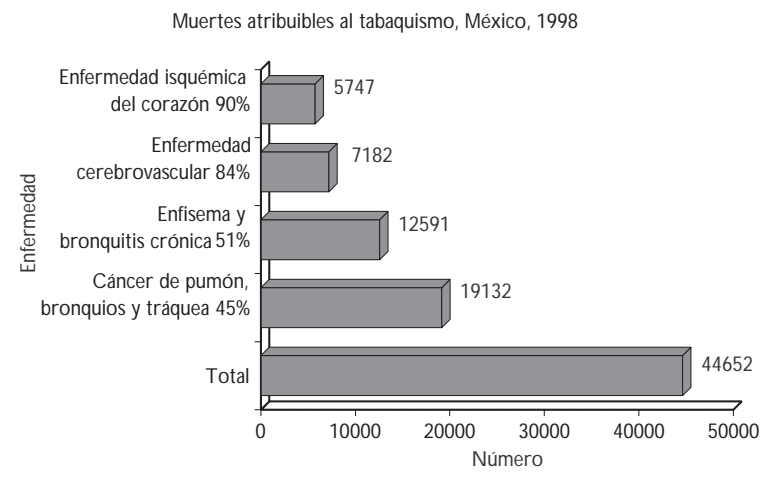

N ota: ( ) = porcentaje de riesgo atribuible

Fuente: Dirección General de Epidemiología/Secretaría de Salud, 1998

Figura 3. Muertes atribuibles al tabaquismo. MéxiCo, 1998

188\% (cuadro I). Si bien existe un incremento en la esperanza de vida en este periodo, que pudiera explicar de manera directa el aumento de estas enfermedades crónicas no transmisibles, se conoce además un incremento del tabaquismo, con un mayor número de muertes por enfermedades atribuibles a su consumo y que se pudieron evitar disminuyendo el tabaquismo.

La cesación disminuye el riesgo cardiaco de cáncer pulmonar y de muerte prematura, de tener hijos de bajo peso al nacer, y mejora la función pulmonar si no hay daño irreparable. Asimismo, disminuye el riesgo de daños a la salud en los fumadores involuntarios. 


\section{Cuadro I \\ MORTALIDAD POR LAS PRINCIPALES CAUSAS DE MUERTE ATRIBUIBLES AL CONSUMO DE TABACO. Méxıco, 1992}

\begin{tabular}{|c|c|c|c|}
\hline Enfermedades 9a CIE & 1970 & 1980 & 1990 \\
\hline Cáncer de pulmón & 1.8 & 3.3 & 5.8 \\
\hline Enfermedad coronaria & 11.9 & 18.6 & 34.3 \\
\hline Enfermedad cerebrovascular & 14.0 & 17.5 & 22.8 \\
\hline EPOC & - & 0.9 & 6.3 \\
\hline O tros cánceres & 3.4 & 4.8 & 7.8 \\
\hline
\end{tabular}

Fuente: referencia 13

No obstante, es importante recalcar que datos de las Encuestas Nacionales de Adicciones indican que el principal motivo para dejar de fumar fue tomar conciencia del daño que ocasiona el tabaco.

\section{Costos: impacto del tabaquismo}

La magnitud del costo asociado con el tabaquismo se observa en los aspectos económicos, así como en el costo de vidas humanas. Los costos directos se estiman con base en la enfermedad causada (gastos en atención médica, atención al enfermo crónico y rehabilitación); la muerte prematura, (pérdida de años de vida productivos y ruptura familiar); el ausentismo laboral, (pérdida de días / hombre de la fuerza laboral) y el ausentismo escolar. De igual forma, la disminución en la calidad de vida de fumadores y fumadores pasivos. ${ }^{14,15}$ En el ámbito familiar de nuestro país, comprar una cajetilla de cigarros equivale al 30\% del salario mínimo diario, lo que a su vez equivale a comprar casi dos litros de leche.

El costo en atención médica se ha estimado en México: en el Instituto Nacional de Enfermedades Respiratorias (INER), en 1994, atender a los pacientes con cáncer de pulmón y enfisema representó $22 \%$ del presupuesto total asignado para la atención médica de dicho instituto para ese año. De igual modo, estimaciones del costo de atención por enfermedades asociadas con el tabaquismo (tumor maligno de tráquea, bronquios y pulmón, enfermedad pulmonar obstructiva crónica, enfermedad isquémica del corazón y enfermedad cerebrovascular) en el Instituto Mexicano del Seguro Social, nos muestran que durante 1994 representó para dicha institución una erogación diaria de más de 194 mil dólares, lo que significó un gasto anual aproximado de 71 millones de dólares. ${ }^{16}$ Podemos inferir que los costos asociados con el consumo de tabaco sobrepasan con mucho las ganancias que produce para el país la industria tabacalera transnacional.

Con el reciente incremento en el impuesto de las cajetillas de cigarros, aprobado por el Congreso en 1999, se espera un aumento en los ingresos de la federación y una consecuente disminución en el consumo per cápita en nuestro país, el cual es actualmente de 754 cigarrillos anuales por persona para el año de 1997. Sin embargo, creemos necesario que parte de estos recursos se destinen a la lucha antitabaco.

Si bien existen tres compañías en el país, prácticamente podemos señalar que el mercado nacional lo comparten dos transnacionales, British American Tobacco (BAT) y Philip Morris. ${ }^{17}$

\section{Prevención y control del tabaquismo}

Las estrategias de prevención y control del tabaquismo en México se han centrado en un punto de vista integral que contempla acciones de:

- prevención,

- protección de los no fumadores,

- cesación y rehabilitación,

- control de la publicidad e

- incremento de impuestos.

De igual modo, se han establecido acciones dirigidas al personal de salud, quienes como líderes de opinión deben buscar la cesación de esta adicción en sus pacientes, así como promover estilos de vida saludables al no fumar y recomendar no hacerlo. Sin embargo, en México la prevalencia de fumadores en el grupo de médicos es más elevada que en la población general, lo cual demuestra que no han asumido el papel que les corresponde en la prevención y control de esta adicción. ${ }^{18-21, *}$

Por otra parte, se ha hecho énfasis en promover el liderazgo de los maestros como ejemplo y lograr que las escuelas sean lugares libres de humo de tabaco. Resulta importante señalar que es necesario enfocar la información sobre los daños causados por fumar en los adolescentes y niños, así como promover estilos de vida saludables. ${ }^{15}$

\footnotetext{
* Instituto Nacional de Enfermedades Respiratorias. Prevalencia de fumadores en los institutos nacionales de salud (INSALUD). México, D.F.: INSALUD, 1997. Documento no publicado.
} 
Asimismo, se han realizado convenios para proteger a los no fumadores, como el suscrito con aerolíneas nacionales para lograr que sus vuelos estén libres del humo de tabaco. Se realizan acciones en el ámbito de legislación, donde se han señalado nuevas restricciones en cuanto a la publicidad y promociones del tabaco para evitar que éstas se dirijan a menores de edad, y se estableció recientemente que dicha publicidad sólo pueda transmitirse en televisión y radio a partir de las 22 horas, y en cines, en películas clasificadas para adultos. Además de vigilar el cumplimiento de la legislación vigente, se consideró eliminar las máquinas autoexpendedoras de cigarros en lugares frecuentados por menores de edad, se prohibió la venta de tabaco por autoservicio y sueltos, y se ha mantenido un control del contenido de los productos del tabaco, obligándolos adicionalmente a señalar en su cajetilla el contenido de nicotina y alquitrán. De igual modo, el 31 de mayo de 2000 se modifica la Ley General de Salud con el propósito de proteger la salud de los no fumadores, lo que ocasiona que se publique en el Diario Oficial de la Federación del 27 de julio del mismo año el Reglamento sobre Consumo de Tabaco, donde se establece con mayor precisión la prohibición de fumar en los edificios públicos. También se señaló que en lugares como cines, teatros, restaurantes, se destinen áreas libres de tabaco. ${ }^{22}$

Así, el 3 de agosto del mismo año, se replantea y refuerza el Programa contra el Tabaquismo, con la finalidad de encauzar las acciones de manera primordial hacia la prevención y control de esta adicción, considerando, además, un sistema que permita evaluar y supervisar de manera integral dicho programa. ${ }^{23}$ Sin embargo, es necesario enfatizar que la responsabilidad y los esfuerzos en la lucha contra el tabaquismo deberán ser compartidos, ya que sólo con una participación de los diversos sectores de la sociedad puede ser remontada esta epidemia.

\section{Conclusión}

En los últimos años existen diversas recomendaciones emitidas por la Organización Mundial de la Salud para combatir esta epidemia y así lograr una reducción en el número de muertes proyectadas para los próximos 20 años. Entre estas recomendaciones destaca el detener el consumo de tabaco, reconocer que la nicotina es una sustancia dañina y psicoadictiva, que el control del tabaco sea una prioridad para los gobiernos y que se impulsen medidas legislativas que tiendan a incrementar las restricciones publicitarias y comerciales del producto, además de buscar una mayor carga fiscal al mismo e incrementar la participación de los países en desarrollo en estrategias globales contra el tabaquismo.

México ha demostrado en los hechos su determinación para impulsar cada una de ellas de manera coordinada y coherente, lo cual se refleja en las decisiones adoptadas en los últimos años.

Sin embargo, debemos señalar claramente la preocupación por el incremento del consumo de este producto por parte de nuestros adolescentes y nuestras mujeres, lo cual se refleja en el análisis de las Encuestas Nacionales, por lo que resulta esencial crear una mayor concienciación y una cultura de prevención, principalmente en estos grupos y entre la población, con el fin de disminuir el consumo de tabaco y, por ende, los daños a la salud que su consumo produce.

Alcanzar esto depende de la participación activa de todos: gobiernos, organizaciones internacionales y no gubernamentales, comunidades e individuos; sólo así podremos legar a nuestros hijos un mundo libre del humo del tabaco.

\section{Referencias}

1.W orld Health 0 rganization. The smoking epidemic- A fire in the global village. 25th August, Ginebra: 1997, Press Release W HO /61.

2. 0 rganización Panamericana de la Salud/O rganización Mundial de la Salud. Tabaco 0 salud: situación en las A méricas. W ashington, D.C.: O PS/O MS, 1992; Publicación Científica N úm. 536.

3. Bradbury J.Annual tobacco-related deaths in China top the half million mark. Lancet 1997; 350:643.

4.W orld Health 0 rganization. The world health report 1996: Fighting disease, fostering development. G inebra:W HO, 1996.

5. Rubio-Monterde $\mathrm{H}$, coord. Contribuciones del Instituto $\mathrm{N}$ acional de Enfermedades Respiratorias en investigación neumológica. Gac Med Mex 1993; 129: 35-51.

6. Secretaría de Salud, Dirección General de Epidemio logía. Encuesta N acional de Adicciones 1993 (EN A-93). México, D.F.: SSA, 1993.

7. Secretaría de Educación Pública, Instituto Mexicano de Psiquiatría. Encuesta $\mathrm{N}$ acional sobre el Uso de Drogas entre la Comunidad Escolar. México, D.F.: SEP, 1992.

8. Kuri-Morales $P, C$ ravioto $P$, Hoy M, Huerta $S$, Revuelta $A$, Jasso $B$ et al. Illegal sales of cigarettes to minors-Mexico City, Mexico 1997. MMW R Morb Mortal W kly Rep 1997; 46: 440-444.

9. Adame-Moreno R, Ibarra O, Torres H, Kuri-Morales P, Hoy M, TapiaC onyer $\mathrm{R}$ et al. Illegal sales of cigarettes to minors- C iudad Juárez, México; El Paso Texas and Las Cruces, N uevo Mexico, 1999. MMW R Morb Mortal W kly Rep 1999; 48: 394-398.

10. Secretaría de Salud, Dirección General de Epidemiología. Sistema de Vigilancia Epidemiológica de Adicciones, Centros de Integración Juvenil. México, D.F.: SSA, 1991-1996.

11. Laniado-Laborín R, Molgaard C, Elder J. Efectividad de un programa de prevención de tabaquismo en escolares mexicanos. Salud Publica Mex 1993;35:403-408.

12. Secretaría de Salud. Mortalidad 1998. México, D.F.:SSA, 1998.

13. Tapia-C onyer R, Kuri-Morales P, Meneses-G onzález F. Smoking attributable mortality-Mexico 1992. MMW R Morb Mortal W kly Rep 1995; 44:372-381. 
14. Departamento de Salud y Servicios Sociales de los Estados Unidos de América. Tabaquismo y salud en las A méricas. Atlanta (GA): D H HS, 1992; Publicación N 0. (CDC) 92-8420.

15. International Union A gainst Tuberculosis and Lung Disease, Tobacco Prevention Section. Educating medical students about tobacco: Planning and implementation. París: Ed. Robyn L. Richmond, 1996.

16. Peña-C orona MP, Mondragón MR, D íaz-Mejía G. Estimación del costo de la atención por enfermedades asociadas al tabaquismo. Higiene 1997;5(1y 2):16-21.

17. Corrao M, Guindon $E$, Sharma N, ed. The $11^{\text {th }}$ World Conference on Tobacco O R H ealth.Tobacco Control C ountry Profiles.A tlanta, G eorgia, agosto 2000.

18. Lara MA, Balzaretti M, $O$ ñate MG, Gómez P. $O$ piniones, actitudes y consumo de tabaco en una institución de salud pública. Salud Publica Mex 1984;26:122-129.
19. Ramírez-Casanova ME, González E, 0 campo A, Sánchez S, Cicero R. Hábito tabáquico entre trabajadores de un hospital general. Informe de una encuesta. Gac Méd Mex 1991; 127:283-288.

20.Tapia-C onyer R, Cravioto P, De la Rosa B, Galván F, García-de la Torre $G$, Kuri P. Cigarette smoking; knowledge and attitudes among Mexican physicians. Salud Publica Mex 1997;39:507-512.

21. D avis R.W hen doctors smoke. Tobacco Control 1993; 2: 187-188.

22. Reglamento sobre el consumo del tabaco. Diario 0 ficial de la Federación 27 de julio 2000, México.

23. Consejo $\mathrm{N}$ acional contra las A dicciones. Programa contra el tabaquismo, México, D.F.: Secretaría de Salud, 2000. 